\title{
Risk-based Causal Modeling of Airborne Loss of Separation
}

\author{
Steven C. Geuther ${ }^{1}$ and Ann T. Shih Ph.D. ${ }^{2}$ \\ NASA Langley Research Center, Hampton, VA, 23681
}

\begin{abstract}
Maintaining safe separation between aircraft remains one of the key aviation challenges as the Next Generation Air Transportation System (NextGen) emerges. The goals of the NextGen are to increase capacity and reduce flight delays to meet the aviation demand growth through the 2025 time frame while maintaining safety and efficiency. The envisioned NextGen is expected to enable high air traffic density, diverse fleet operations in the airspace, and a decrease in separation distance. All of these factors contribute to the potential for Loss of Separation (LOS) between aircraft. LOS is a precursor to a potential mid-air collision (MAC). The NASA Airspace Operations and Safety Program (AOSP) is committed to developing aircraft separation assurance concepts and technologies to mitigate LOS instances, therefore, preventing MAC. This paper focuses on the analysis of causal and contributing factors of LOS accidents and incidents leading to MAC occurrences. Mid-air collisions among large commercial aircraft are rare in the past decade, therefore, the LOS instances in this study are for general aviation using visual flight rules in the years 2000-2010. The study includes the investigation of causal paths leading to LOS, and the development of the Airborne Loss of Separation Analysis Model (ALOSAM) using Bayesian Belief Networks (BBN) to capture the multi-dependent relations of causal factors. The ALOSAM is currently a qualitative model, although further development could lead to a quantitative model. ALOSAM could then be used to perform impact analysis of concepts and technologies in the AOSP portfolio on the reduction of LOS risk.
\end{abstract}

\section{Introduction}

$\mathrm{T}$ HE U.S. National Airspace System (NAS) is arguably one of the safest in the world today. However, there remain aviation challenges in some key areas, one of which is airborne losses of separation ${ }^{1}$. Losses of separation (LOS) continue to be a major aviation safety concern as the current U.S. Air Transportation System (ATS) transforms into the NextGen operating environment with the forecast of a significant increase in air traffic density $^{2}$, complexity of operations, and diversity of a fleet mix including the emerging market of the GA fleet for on-demand mobility. In synergy with the Federal Aviation Administration (FAA) advancing the NextGen ATS, the National Aeronautics and Space Administration's (NASA's) Airspace Operations and Safety Program (AOSP) aims at creating technologies to achieve high-impact, system-wide operational benefits for the NextGen in increasing capacity and reducing cost, while maintaining safety and efficiency. Among many NextGen systems improvements, the implementation of reduced/new separation standards ${ }^{2,3}$ is proposed as a way toward increasing capacity and reducing delays. Therefore, understanding the past LOS instances and addressing the potential future LOS become critical for a better assessment of the contribution of the NASA AOSP's research and development activities to aircraft separation assurance.

Losses of required separation occur when aircraft fail to maintain the separation minima set forth by the FAA. When separation is lost, it increases the chance of a mid-air collision, but does not often result in the aircraft colliding. The likelihood of a collision is reduced due to a considerable safety buffer built into the safety standards. This generally provides the pilot or controller ample time to resolve the situation before it becomes critical. As a last resort, the Traffic Collision Avoidance System (TCAS) is designed to prevent collisions for aircrafts that have this

\footnotetext{
${ }^{1}$ Student Engineer, Aeronautics Systems Analysis Branch, E-mail: steven.c.geuther@nasa.gov

${ }^{2}$ Senior Researcher, Aeronautics Systems Analysis Branch, E-mail: ann.t.shih@nasa.gov

1

American Institute of Aeronautics and Astronautics
} 
technology. Aircraft separation, however, is still a complex operation with many layers of measures, in which a LOS event can occur and the early detection and proper correction of these losses may mitigate against aircraft collisions.

The objective of the research presented in this paper is to analyze LOS accidents and incidents with an emphasis on General Aviation (GA) using visual flight rules (VFR), not only identifying the causal and contributing factors to airborne LOS, but also the relations of these factors. The relations are modeled using Bayesian Belief Network ${ }^{4}$ (BBN) technique. The model is called the Airborne Loss of Separation Analysis Model (ALOSAM). The ALOSAM is a high-level generalized model comprising causal factor analysis results of both LOS accidents and LOS incidents for the time period of 2000-2010. This model provides a graphical depiction of interrelated active causes and latent causes, leading to the occurrence of LOS/MAC. The ALOSAM can be used for performing impact analysis of concepts and technologies in the AOSP portfolio on the risk reduction of LOS leading to mid-air collisions.

\section{LOS/MAC Accidents/Incidents Database Review}

When investigating the mid-air collision occurrences from LOS, the accidents and incidents database from the National Transportation Safety Board (NTSB) and the Aviation Safety Reporting System (ASRS) were used, respectively. The instance search was for the years span from 2000 to 2010 . The years of choice allowed for all instances to have a finalized report completed. Over this 11 year span, there were 15 incidents and 87 accidents, which came mostly from general aviation. The LOS instances can be categorized based on the flight phase in which the LOS occurs. These flight phases are: standing/taxing, takeoff, climb, cruise, descent, approach, landing, goaround, and maneuvering. ${ }^{5}$ For the incidents, cruise accounted for $54 \%$, climb for $13 \%$, descent for $13 \%$, and approach for $20 \%$. For the accidents, cruise accounted for $23 \%$, climb for $9 \%$, descent for $3 \%$, approach for $33 \%$, standing/taxing for $1 \%$, takeoff for $3 \%$, landing and go-around for $2 \%$ each, and maneuvering for $24 \%$. The distribution can be seen in Figs. 1 and 2. The incident statistics show that the cruise phase is the most prevalent, but this is for a small amount of events. While from accident statistics, the most significant flight phase is seen to be approach over a greater amount of events than incidents and, therefore, becomes the phase of interest. If LOS can be prevented in this one phase of flight, there will be a large decline in total LOS instances due to a decrease in the primary occurrence phase. In this study, the approach phase is then combined with go-around, landing, and descent as all three of these instances have minimal cases and are correlated with the last part of flight. Due to the insignificant commercial LOS instances leading to MAC, the study is reduced from 5 incidents and 38 accidents to 4 incidents and 35 accidents for a further detailed study by removing non-GA events. These chosen 39 LOS instances for causal study in Section III \& IV are GA operations with VFR. 


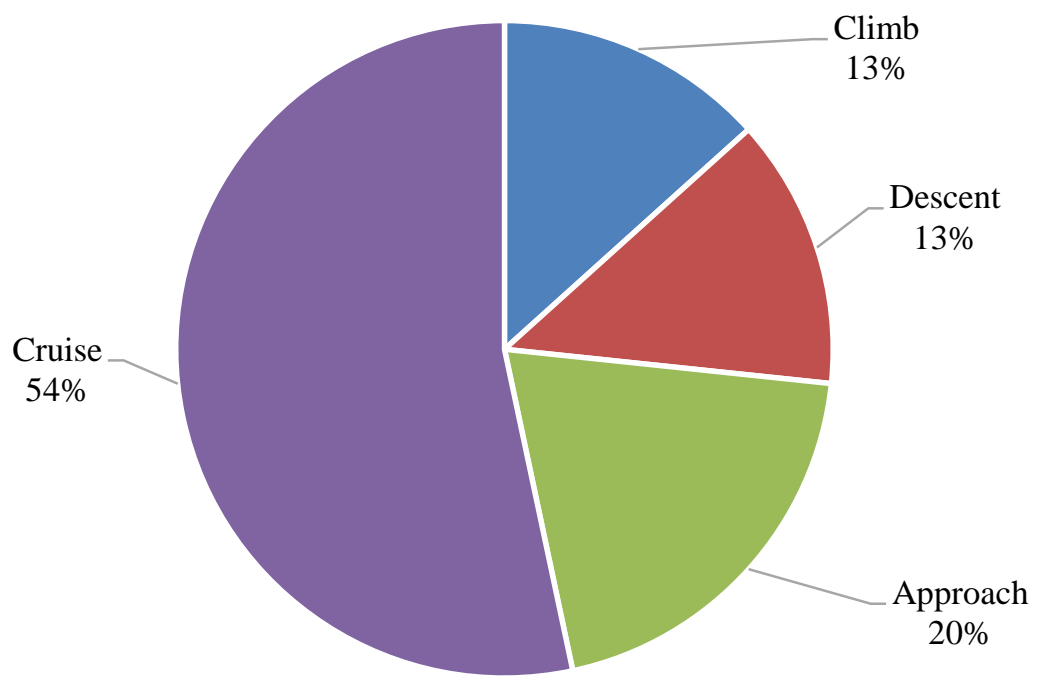

Figure 1. LOS incident phase distribution.

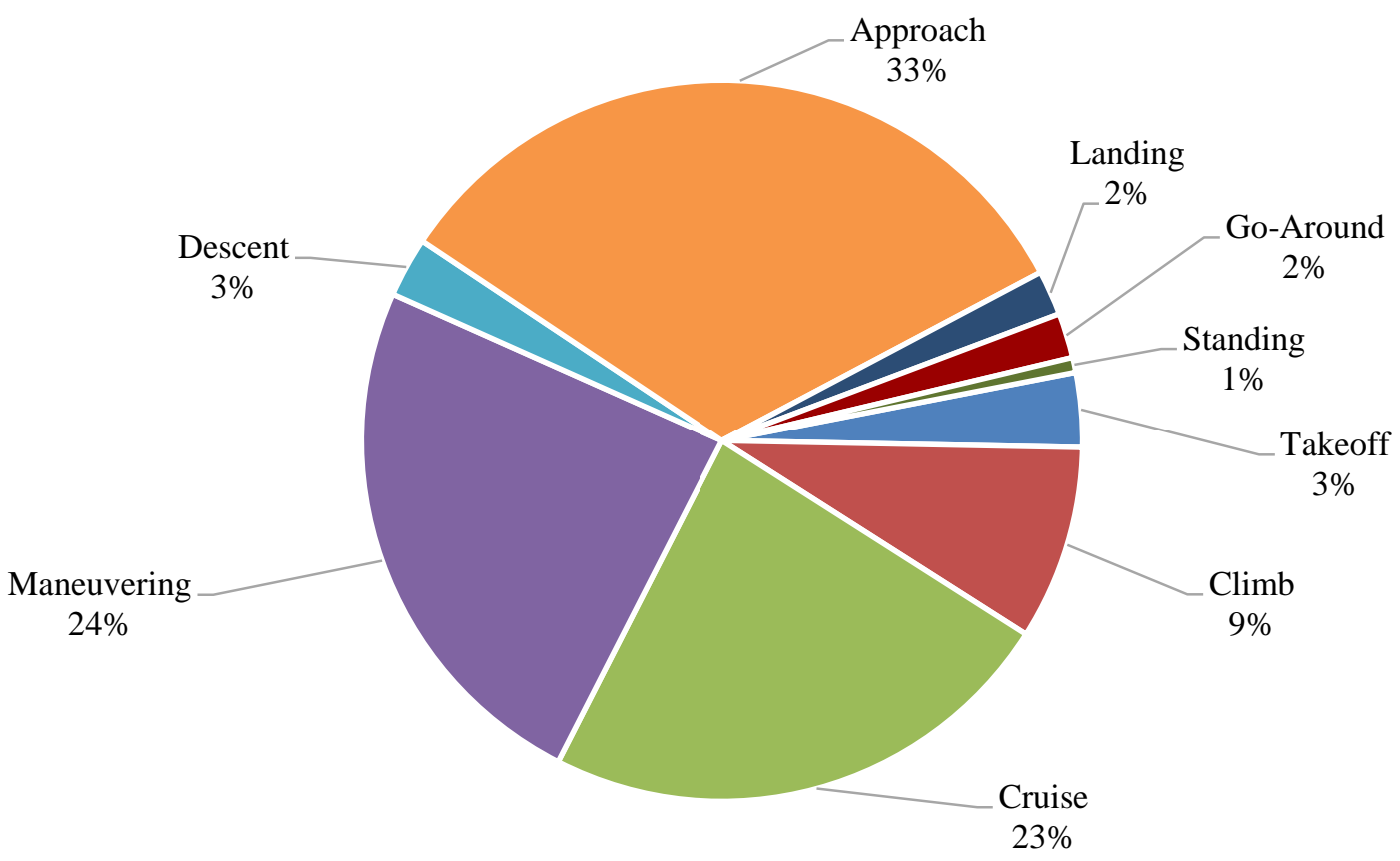

Figure 2. LOS/MAC accident phase distribution.

The FAA in recent years has collected data on the number of risk analysis events (RAE) referring to LOS for their air traffic organization safety reports ${ }^{6}$. Risk analysis events are categorized from all validated LOS occurrences, where less than two-thirds of the required separation is maintained. High-risk LOS events are then selected from the RAE based upon a combination of likelihood and severity. The likelihood category is split into 5 categories ranging 
from extremely improbable to frequent. For a RAE to be classified as high-risk, the likelihood must be probable or greater, where probable is defined as the event is expected to occur often ${ }^{7}$. The severity category is also split into 5 categories ranging from minimal to catastrophic. A high-risk event is a result of a severity of major or greater, meaning there is substantial damage to the aircraft and/or physical distress or injuries to the people aboard ${ }^{7}$. Over the 2012 fiscal year there were 41 high-risk events, with a slight decrease in 2013 of 38 events achieving a rate of 5.66 high-risk losses per thousand. Although this is below the FAA strategic goal maximum occurrence of 20 high-risk events for every 1,000 LOS events ${ }^{6}$, it is desirable to eliminate as many high-risk events as possible as this is still perceived as a safety issue. However, the aircrafts that are included in this data cannot be differentiated as commercial or GA or as flights that use visual flight rules or instrument flight rules.

In addition, the Bureau of Transportation Statistics (BTS) in U.S. Department of Transportation provides a number count of Near Midair Collisions (NMAC) by degree of hazard from pilot reports from 1980 to $2013^{8}$ for all flights. These NMAC instances are reported voluntarily to the FAA, implying they may not be totally representative, but providing some interesting observations as shown in Fig. 3. The degree of hazard of critical listed in Fig. 3 is defined as aircraft separation of less than 100 feet, where collision avoidance is due to chance rather than an act of the pilot. ${ }^{8}$ Potential refers to an aircraft separation of less than 500 feet that would probably result in a collision if no action was taken by a pilot, while no hazard indicates a difference in direction or altitude, which makes the LOS improbable with or without an evasive action. ${ }^{8}$ Finally, an unclassified LOS has insufficient evidence to classify the LOS in the aforementioned categories. ${ }^{8}$ Since 1990, the general trend of reported NMAC instances appears to decline up to 2009, and then a gradual increase afterwards. Similarly to the FAA RAE, it is not clear what type of aircraft or type of flight rules are used for each instance in BTS database. The occurrence of LOS is very much impacted by the factors of pilots, air traffic controllers, and the flight demands. Figures 4-6 show the statistics of the number of active pilots ${ }^{9}$, demand of domestic flights ${ }^{10}$, and the trend of ATC hires and losses ${ }^{11}$ over a similar time period. All three trends may be related to the NMAC trend and will be of interest as the number of new pilots and demand continue to gradually grow and the number of ATC employees and experienced pilots decrease since 2009. 


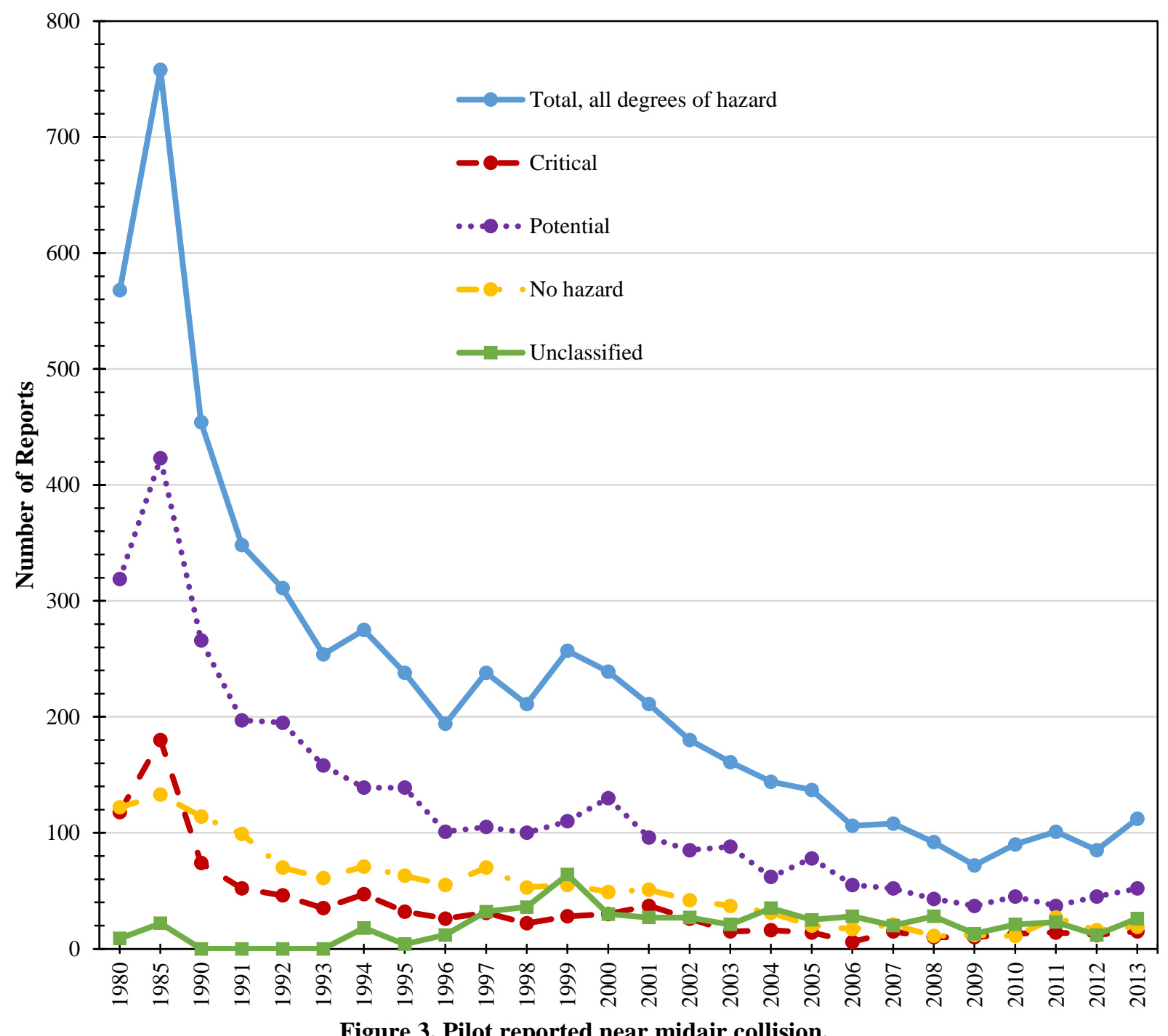

Figure 3. Pilot reported near midair collision. 


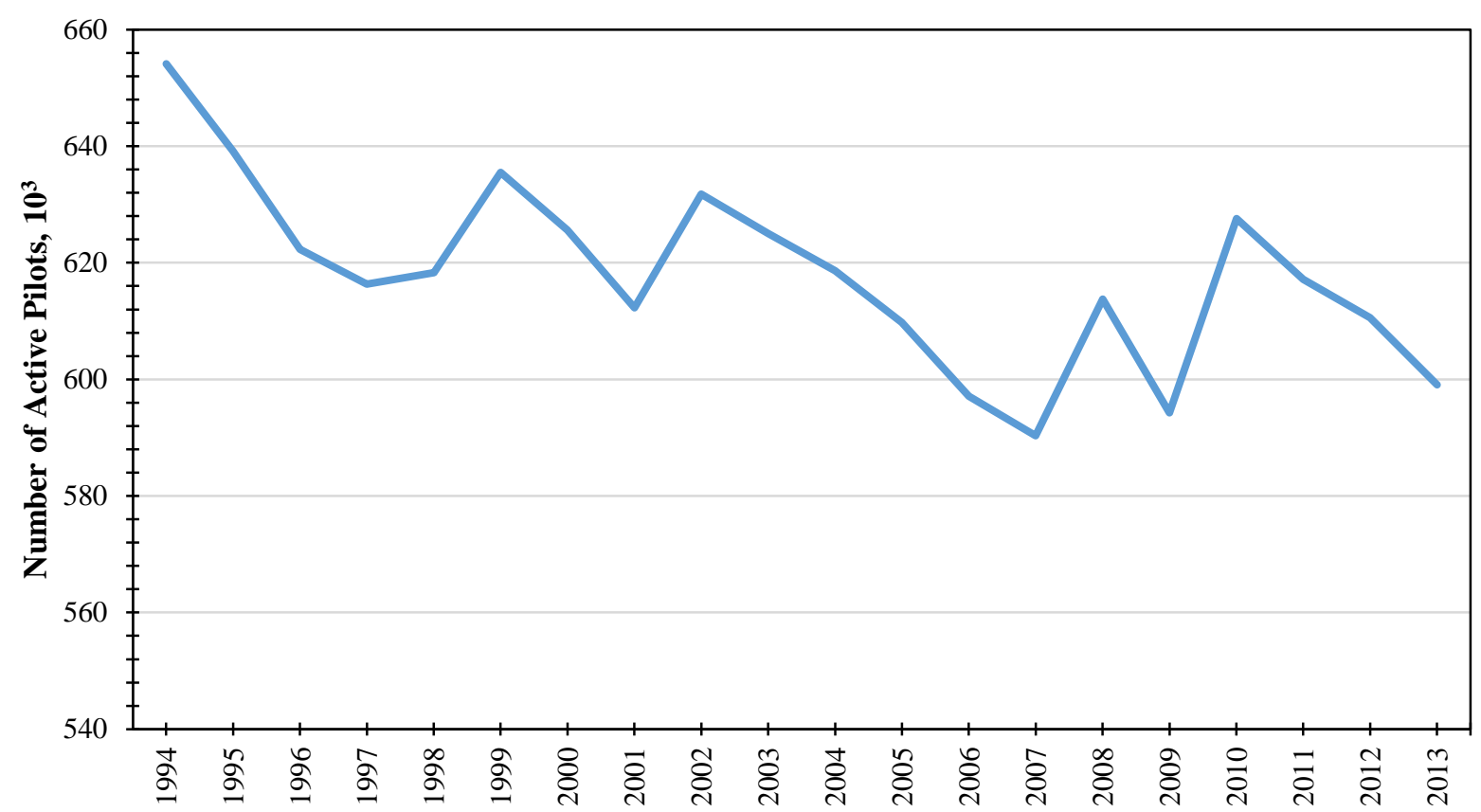

Figure 4. Active pilots timeline.

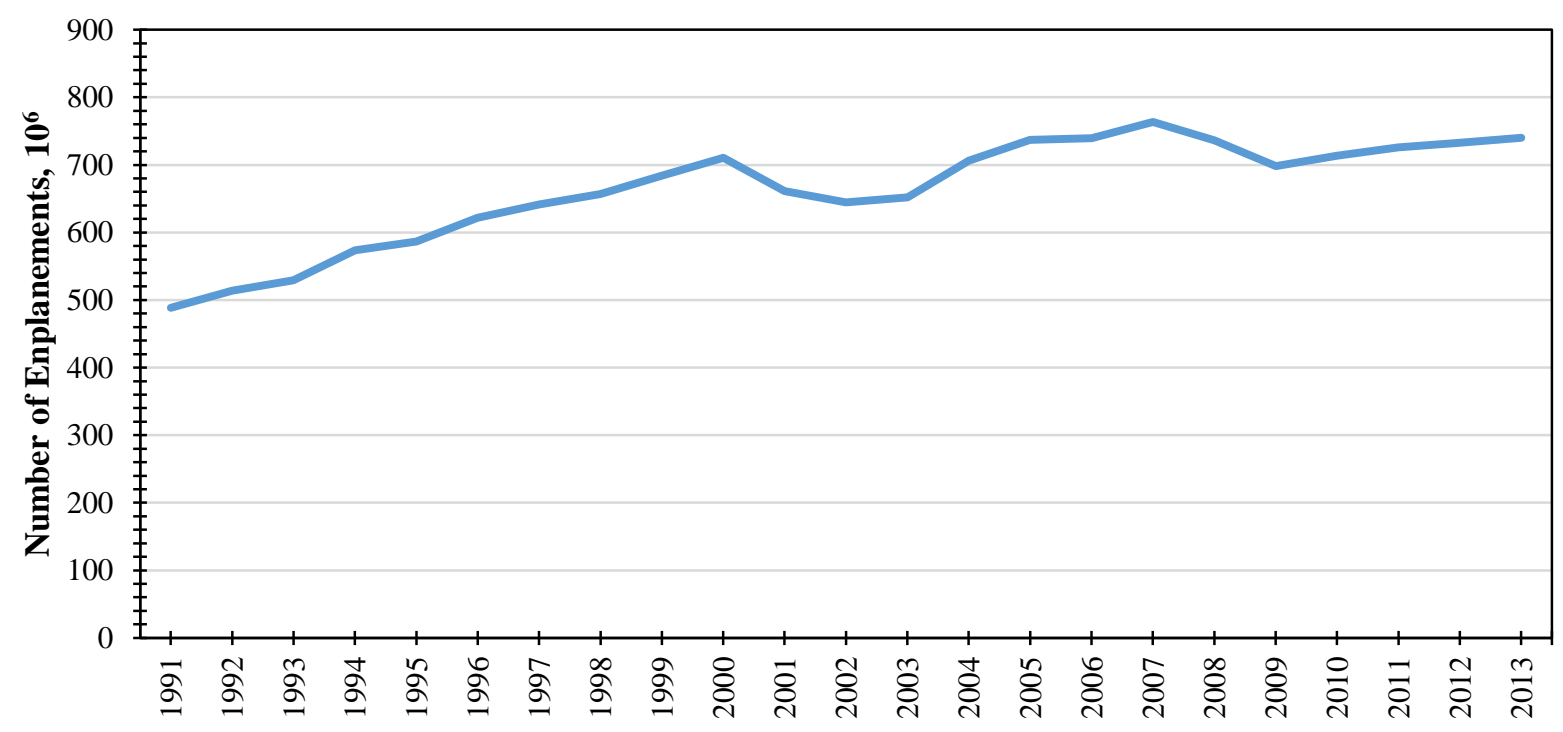

Figure 5. Total number of passenger enplanements timeline. 


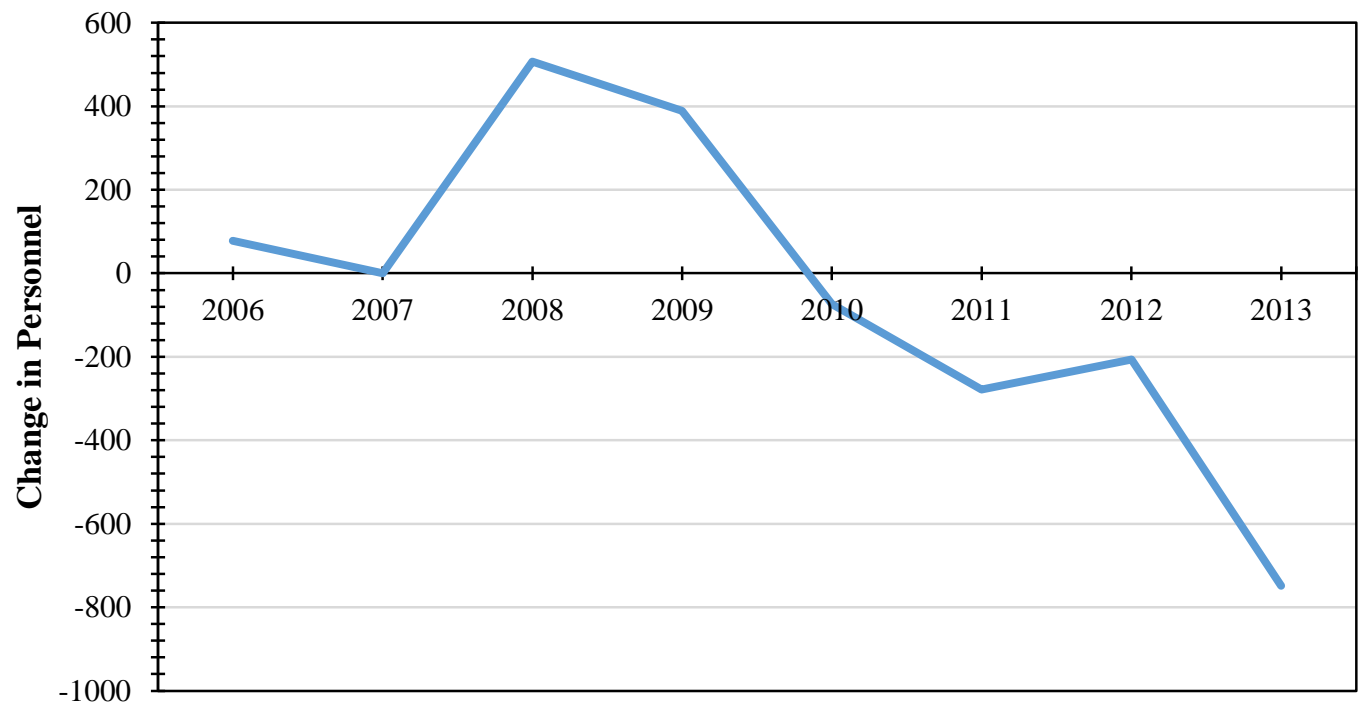

Figure 6. Net ATC personnel timeline.

\section{Anatomy/Analysis of LOS Causal Factors}

After reviewing all of the chosen LOS instances for the descent, approach, landing, and go-around phases, causal factors were identified from the report narratives, both explicitly and implicitly. It was determined that there are 5 different causal factors for the LOS incidents and 23 for the LOS accidents. This allowed for a correlation analysis and factor count to be performed to observe trends for the selected phases. The correlation analysis was performed through a function in excel to help build a model to be discussed in part IV. A correlation analysis defines a measure of association between causal factors and, therefore, the links connecting the factors to each other. The factor count was created based upon each individual phase and the combined phases in order to see if there was a more prevalent causal factor in a select phase compared to the overall trend.

The main influences for LOS to occur as an incident were determined to be from the pilot lacking equipment, pilots and Air Traffic Controller(s) (ATC) not communicating, ATC situational awareness, and pilot visual awareness. The factor count for all considered flight phases is shown in Fig. 7. However, both the correlation analysis and the factors count for the incidents were determined not sufficient for making future assumptions due to the small sample size with only 4 instances in review.

With 35 accident reports, the correlation analysis and causal factor count have some significance. The factor count shown in Fig. 8 indicates how many times each of these 23 different causal factors occurred throughout the 35 instances for all considered phases. It should be noted that all of the causal factors that occurred in the incidents also appeared in the accidents but at different occurrence rates for a proportional amount of instances. The total frequency count results indicate that the pilot visual awareness is the top causal factor in LOS/MAC accidents. This result correlates with GA operations with VFR while these accidents occurred. This is seen as a similar result in the LOS/MAC incidents. The trend of the factor count for each of the individual phases, shown in Figs. 9 and 10, is consistent with the total count, except for the descent phase. The descent phase shows that other causal factors, including pilot health/fatigue and pilot equipment failure are more prevalent than the pilot communication. However, this is not statistically significant because there are very few reports relating to LOS/MAC in the descent phase. 


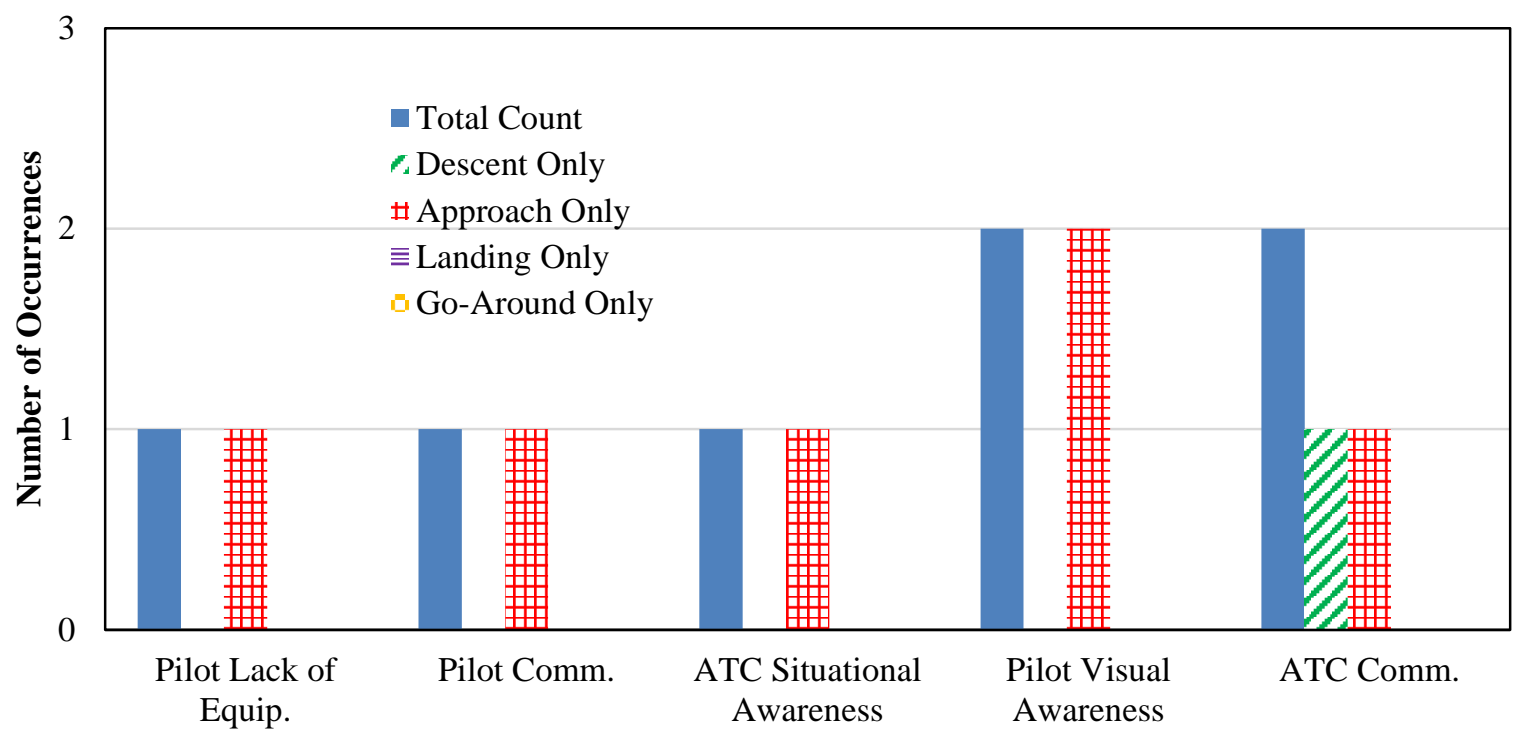

Causal Factor

Figure 7. LOS/MAC incident causal factor count in descent, approach, landing, and go-around phases for GA.

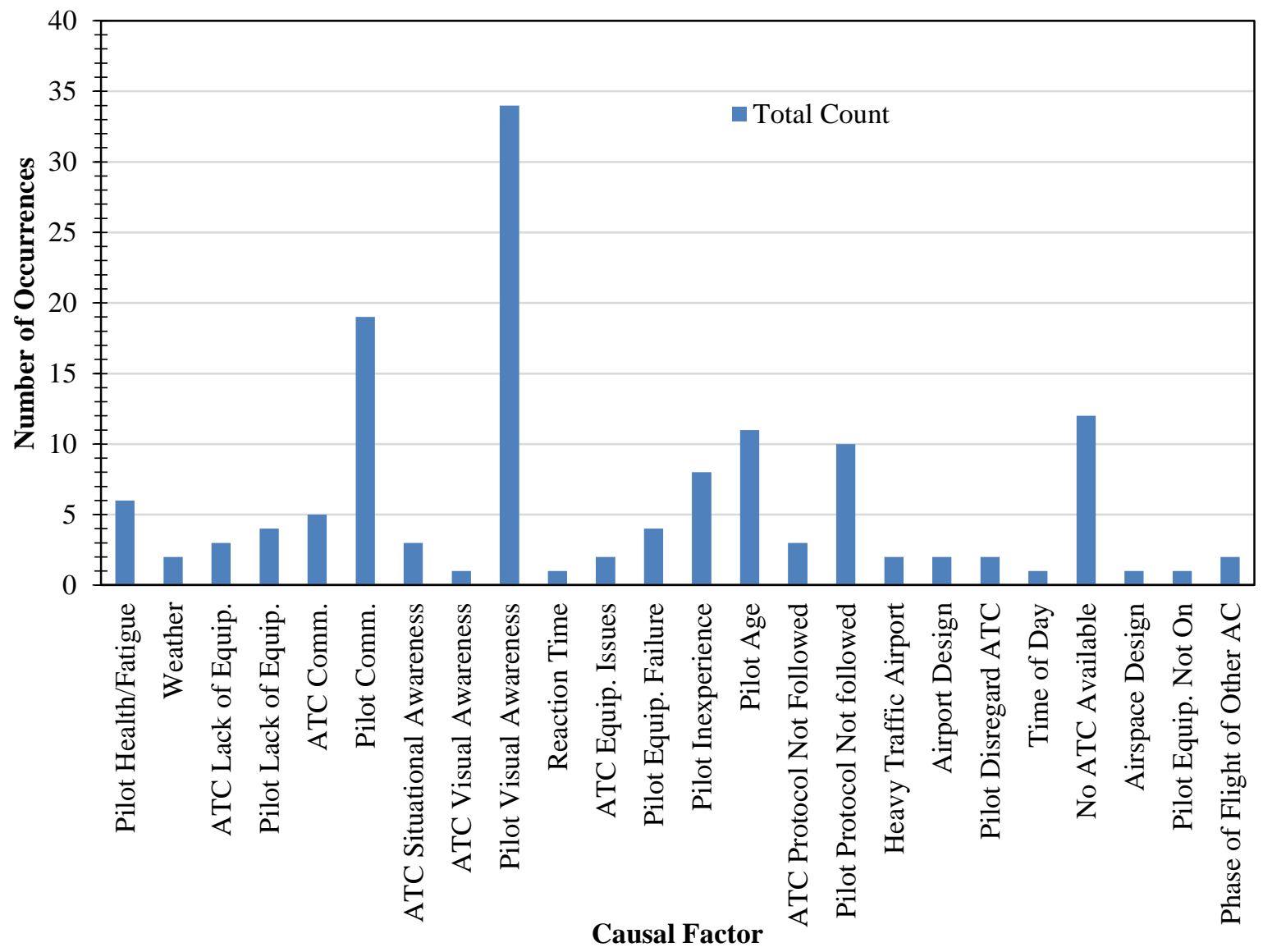

Figure 8. LOS/MAC accident total causal factor count in descent, approach, landing, and go-around phases for GA.

8

American Institute of Aeronautics and Astronautics 


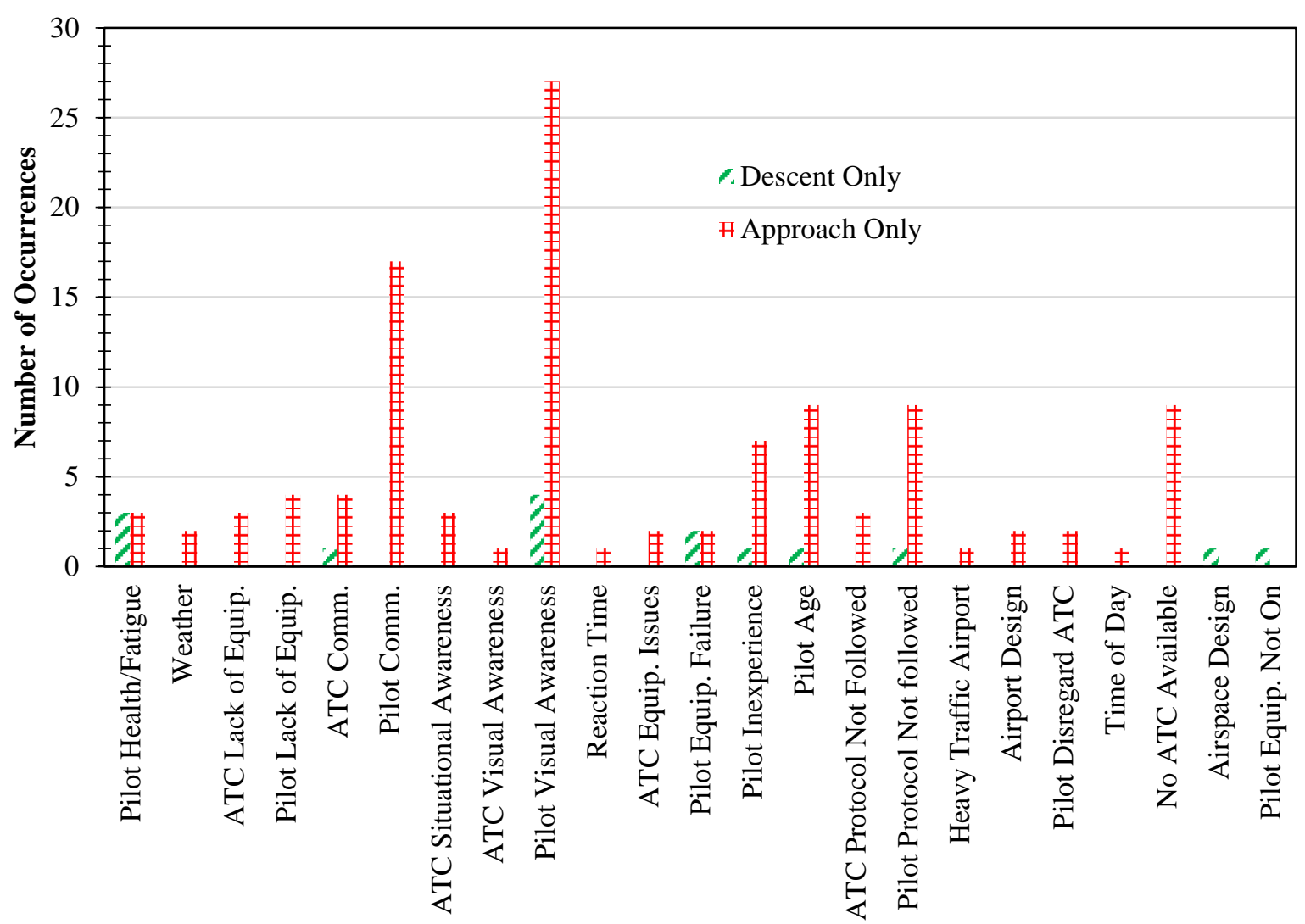

Causal Factor

Figure 9. LOS/MAC accident causal factor count in descent and approach phases for GA.

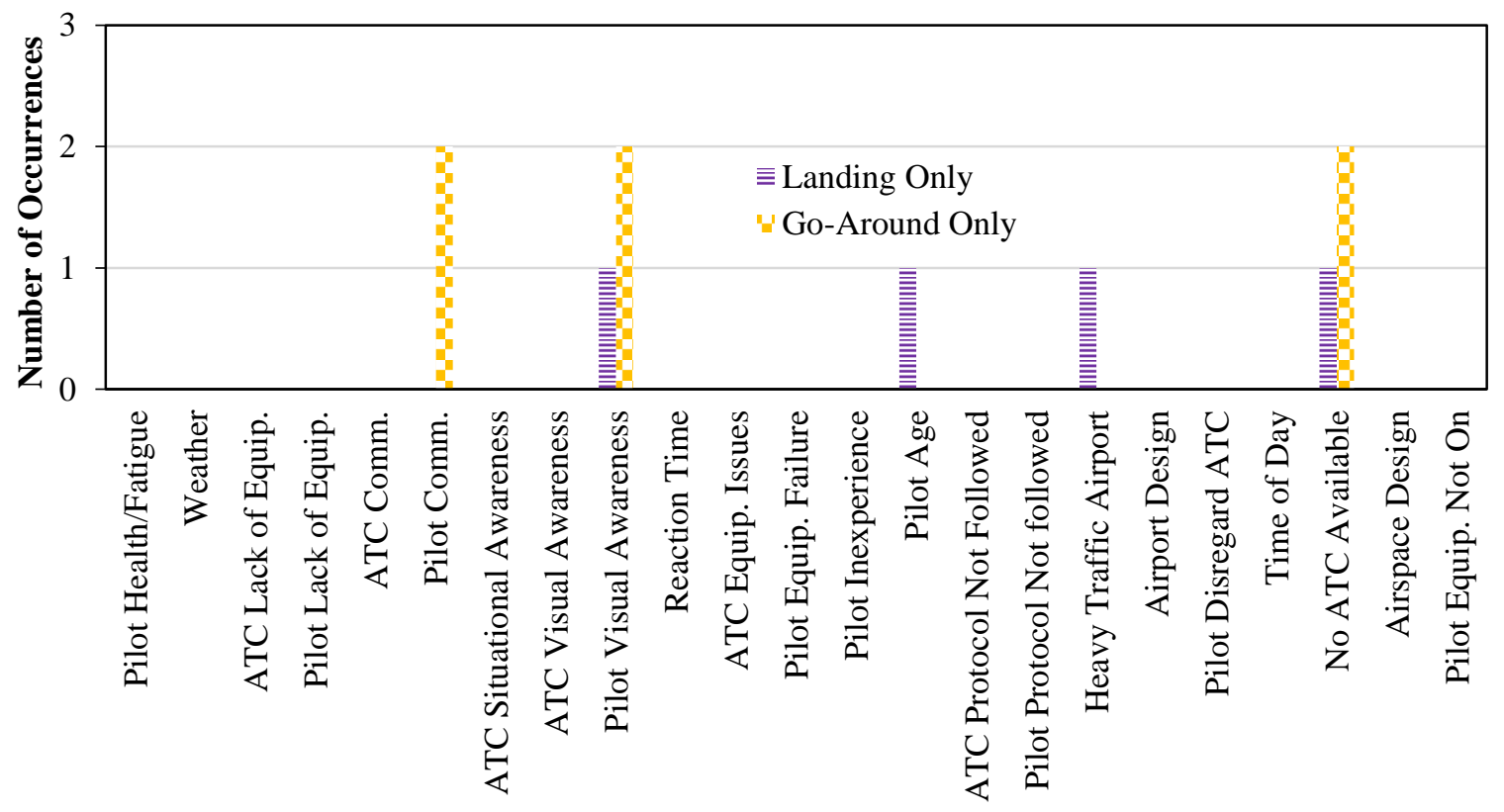

Causal Factor

Figure 10. LOS/MAC accident causal factor count in landing and go-around phases for GA. 


\section{Risk-based Causal Modeling}

The identified causal factors and their relations from the correlation analysis are better captured through a visual representation of cause and effects with multi-dependency. The aspect of multi-dependency is modelled well through the BBN technique using software called Hugin Explorer ${ }^{12}$. The technique is built upon nodes that represent the causal factors and arrows to each node that represent the causal dependency between the two or more factors ${ }^{13}$. A high-level generalized BBN is used to model the LOS incidents and accidents, respectively, as depicted in Figs. 11 and 12. Collectively, the model is called the Airborne Loss of Separation Analysis Model (ALOSAM).

Figure 11 represents a notional BBN for LOS incidents for the four aforementioned phases, which has five causal factors. The BBN for LOS accidents for the same four phases of flight with 23 different causal factors is more involved and complex as seen in Fig. 12. Due to a small number of studies incidents, the BBN in Figure 11 is notional, showing how factors may influence each other and the factors ultimately contribute to the LOS as an incident. Figure 12 is color-coded to show the primary (green), secondary (blue), tertiary (orange), and fourth (pink) order influences on a LOS accident occurrence. The nodes that do not have arrows going into them are unconditioned nodes, and considered independent of influence by other nodes in the model and, therefore, are left in the generic yellow color. As seen in both models, a pilot has the most opportunities for error leading up to a LOS. This suggests that the pilot is being affected by all other operations and influences that go on during flight.

The ALOSAM is currently a qualitative model displaying multiple causal paths leading to the LOS instances. Further development of the ALOSAM can be made to populate the (un)conditional probabilities of each node, allowing for a quantitative, probabilistic analysis of LOS, and assessing the impact of concepts and technologies $(\mathrm{C} / \mathrm{Ts})$ in the AOSP portfolio on the reduction of LOS risk. Typically, this requires several meetings with subject matter experts to review and refine the model structure, to insert the $\mathrm{C} / \mathrm{Ts}$ into the model, and to populate the node probability without and with $\mathrm{C} / \mathrm{Ts}$.
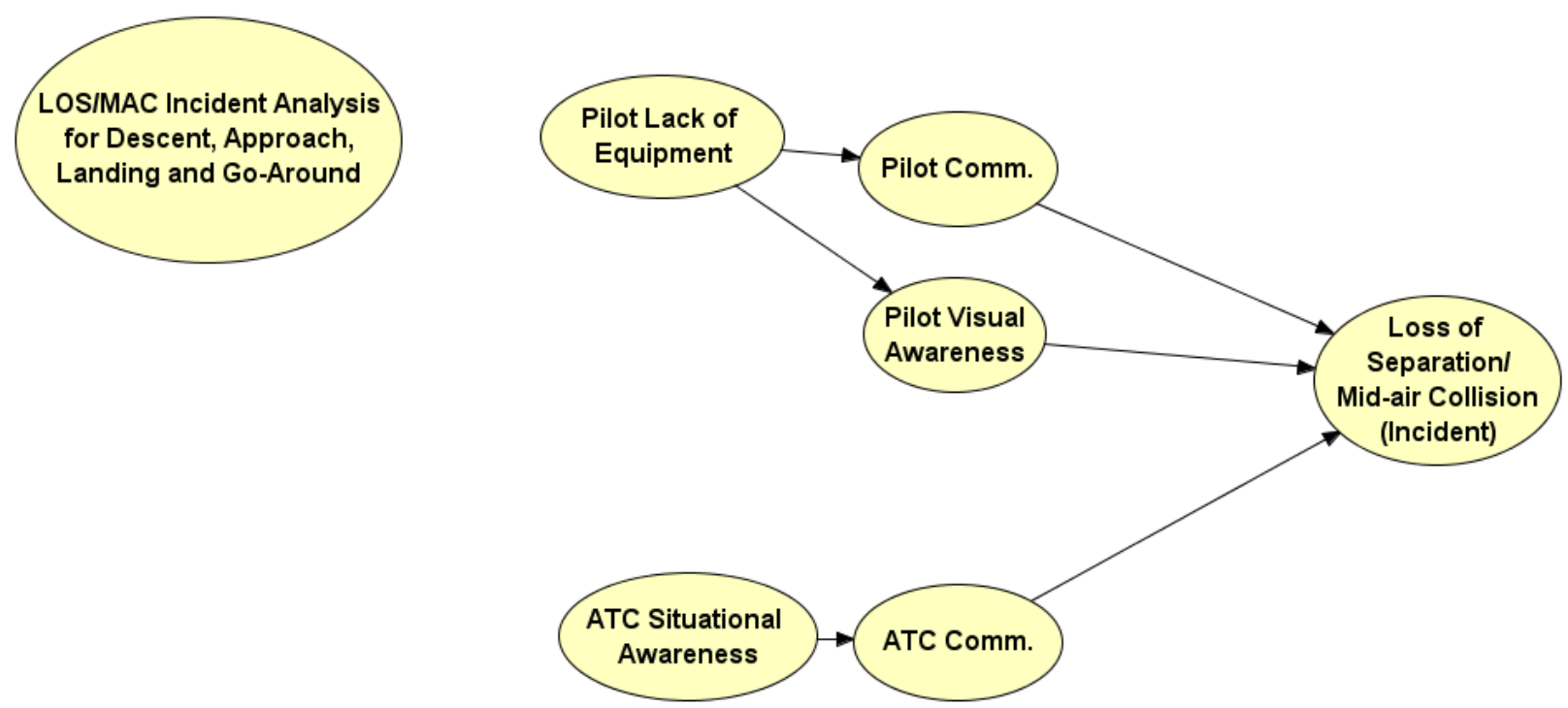

Figure 11. Notional BBN for LOS/MAC incidents for GA. 


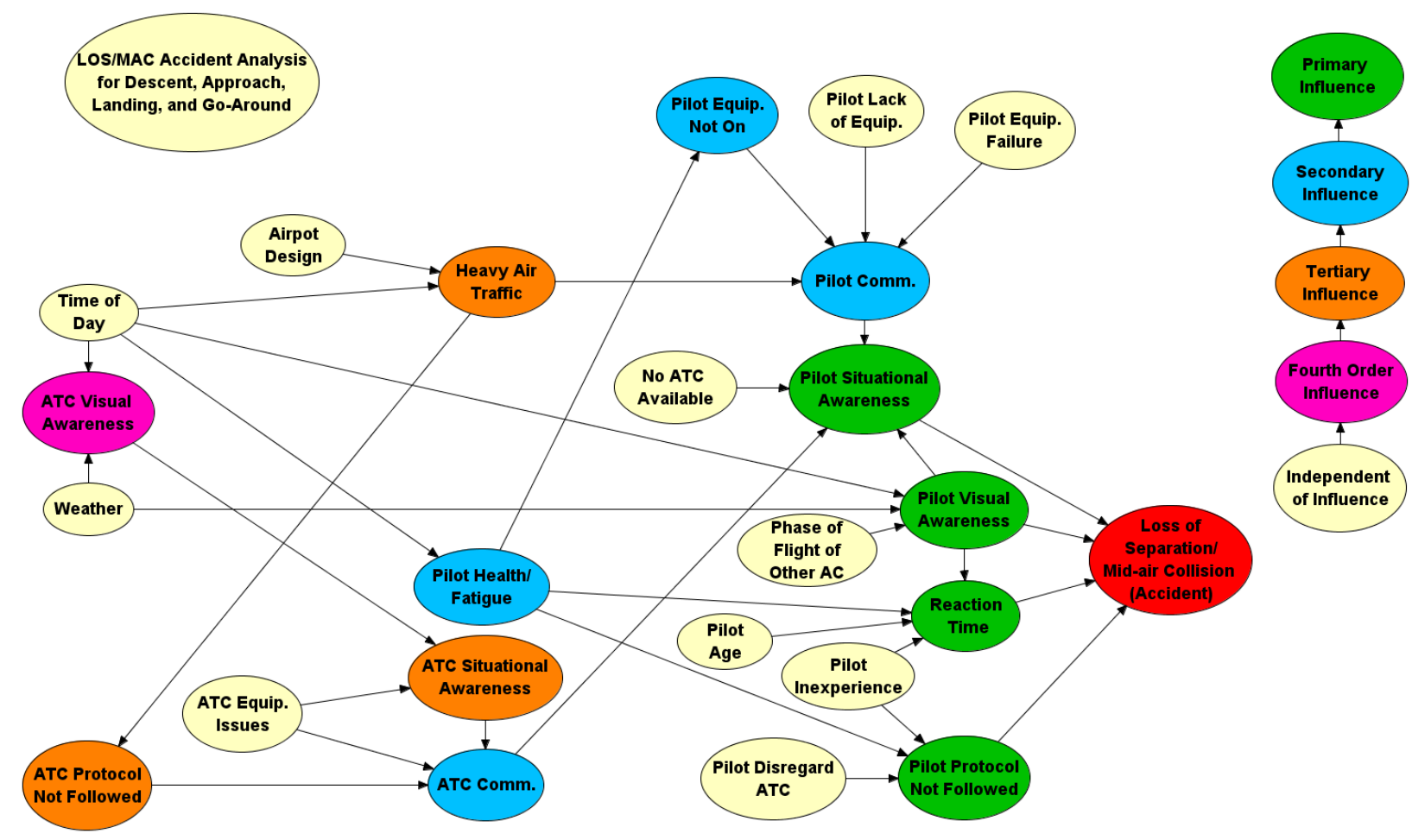

Figure 12. Preliminary BBN for LOS/MAC accidents for GA.

\section{Conclusions}

This paper shows the results of an analysis of LOS instances occurred in four flight phases: descent, approach, landing and go-around in the years 2000-2010. The analysis focuses on general aviation using visual flight rules because LOS of GA aircraft is more likely to lead to MAC than the large commercial aircraft, and the GA fleet is emerging to satisfy the on-demand mobility in the future NAS. The leading causal factors in these LOS instances resulting in MAC were then identified. These factors should be mitigated to promote aviation safety with the upcoming NextGen implementations and demands. The study also includes the development of a preliminary BBN model, in which more than half of the active causal factors for LOS/MAC accident are related to the pilots. With further development, this preliminary model can be made into a quantitative model to evaluate the contribution of NASA technologies on the reduction of LOS risk of GA type of operations. Furthermore, a similar analysis of the other phases of a flight could be established, which will lead to a better impact assessment of technologies on LOS/MAC in a full flight spectrum.

\section{References}

${ }^{1}$ United States Government Accountability Office (GAO), "Aviation Safety: FAA Efforts Have Improved Safety, but Challenges Remain kin Key Areas," GAO-13-442T, Washington, D.C., April 16, 2013.

${ }^{2}$ Joint Planning and Development Office (JPDO), "Concept of Operations for the Next Generation Air Transportation System," Version 2, June 13, 2007.

${ }^{3}$ The Federal Aviation Administration, "NextGen Implementation Plan," June 2013.

${ }^{4} J e n s e n$, Finn V., and Thomas D. Nielsen, Bayesian Networks and Decision Graphs, Springer-Verlag, New York, 2007.

${ }^{5}$ The International Civil Aviation Organization and the Commercial Aviation Safety Team, "Glossary of Terms", http://www.intlaviationstandards.org/apex/f?p=240:1:30075027641970::NO::P1_X:Glossary, Updated 2014 
${ }^{6}$ The Federal Aviation Administration, “Air Traffic Organization 2013 Safety Report,” Published April 30, 2014

${ }^{7}$ The Federal Aviation Administration, "Safety Risk Management Policy,” April 2012

${ }^{8}$ United States Department of Transportation, Bureau of Transportation Statistics, "Table 2-15 - Number of Pilot-Reported Near Midair Collisions (NMAC) by Degree of Hazard,"

http://www.rita.dot.gov/bts/sites/rita.dot.gov.bts/files/publications/national_transportation_statistics/html/table_02_1 5.html, October 2014.

${ }^{9}$ The Federal Aviation Administration, "US Civil Airmen Statstics,"

https://www.faa.gov/data_research/aviation_data_statistics/civil_airmen_statistics/2013/, July 2014.

${ }^{10}$ The Federal Aviation Adminstration, "Passenger Boarding (Enplanement) and All-Cargo Data for U.S.

Airports", http://www.faa.gov/airports/planning_capacity/passenger_allcargo_stats/passenger/, October 2014.

${ }^{11}$ The Federal Aviation Administration, “Air Traffic Controller Workforce Plan 2014-2023," September 2013

${ }^{12}$ Hugin Explorer, V.8.0 (2014), Hugin Expert A/S, Denmark.

${ }^{13}$ Ancel, Ersin \& Shih, Ann T., "The Analysis of the Contribution of Human Factors to the In-flight Loss of Control Accidents," 12 th AIAA Aviation Technology, Integration, and Operations (ATIO) Conference, Indianapolos, IN, Sept 17-19, 2012. 